\title{
Computational Analysis on the Binding of Epitope Peptide to Human Leukocyte Antigen Class I Molecule A*2402 Subtype
}

\author{
MD Iqbal Mahmood, Yuri Matsuo, Saburo NeYa, and Tyuji Hoshino* \\ Graduate School of Pharmaceutical Sciences, Chiba University; 1-33 Yayoi-cho, Inage-ku, Chiba 263-8522, Japan. \\ Received June 15, 2011; accepted July 25, 2011; published online July 26, 2011
}

\begin{abstract}
Immunological response induced by small amino peptide has attracted much recent attention in the field of immunotherapy. Wilms' tumor (WT1) protein is one of the potent tumor antigens inducing immunological response in mouse and human, because WT1 is over expressed in many types of leukemia and various kinds of solid tumors. A 9-mer peptide encoded in WT1 protein (CMTWNQMNL; amino acid 235-243) is known to serve as antigenic peptide for human leukocyte antigen (HLA)-A*2402 molecule. It was reported that the replacement of the second amino residue, which is deeply responsible for the peptide binding to HLA, induced strong immunological response compared to the natural peptide. In this study, 19 kinds of single amino substitutions were introduced at position 2 of this 9-mer WT1 peptide. We performed molecular dynamics simulation on the complex of each of WT1 epitope peptides and HLA- $\beta 2$ micro globulin $(\beta 2 \mathrm{~m})$ molecule, and subsequently estimated the binding affinity using molecular mechanics/generalized-Born surface area method combined with normal mode analysis. Our computation indicated that the peptide containing M2Y or M2W mutation showed high binding affinity to the HLA- $\beta 2 \mathrm{~m}$ molecule as well as the natural peptide. We have also examined the role of the residue at position 2 in peptide binding to HLA- $\beta 2 \mathrm{~m}$. The calculation showed that van der Waals interaction between the side chain of the residue at position 2 and hydrophobic residues inside B-pocket of HLA are important. These findings will be helpful to search other potent peptides that will enhance strong immunological response specific to $\mathrm{HLA}-\mathrm{A} * \mathbf{2 4 0 2}$ molecule.
\end{abstract}

Key words human leukocyte antigen; molecular dynamics simulation; binding affinity; epitope peptide; anchor residue

Major histocompatibility complex (MHC) is a transmembrane glycoprotein that plays an important role in immunological system. Human MHC molecule is usually called as human leukocyte antigen (HLA) and HLA molecules are grouped into class I and II. MHC class I molecule is a heterodimer of heavy chain called $\alpha$ chain, whose mass-weight is $45 \mathrm{kDa}$, and light chain called $\beta 2$ micro globulin $(\beta 2 \mathrm{~m})$ with a mass-weight of $12 \mathrm{kDa}$. A complex of an HLA and a peptide derived from antigen is displayed on the surface of nucleated cell or platelet. HLA heavy chain has a quite large diversity and thousands of genes have been detected so far, and the number of known genes for HLA heavy chain is still increasing. Each individual has two or three kinds of HLA out of several thousands of HLA genes.

Viral protein or cancer-related antigens are detected as foreign molecules in a cell and dissociated by proteasome into peptide fragments consisting of $6-15$ amino residues. ${ }^{1)}$ This peptide fragment is bound to HLA class I molecule and the complex is carried to the cell surface in antigen display cells. Cytotoxic $\mathrm{T}$ lymphocytes (CTL) expressing $\mathrm{T}$ cell receptor recognize the complex, which results in inducing the immunological response to attack the virus-infected or oncogenic cells to exclude virus or tumor. ${ }^{2)}$

Immunological response has attracted much recent attention because of its potential for immunotherapy. At present, several peptides are assumed to be effective for medical treatment of cancer, leukemia, hepatitis $\mathrm{C}$, and applied to patients in a clinical trial as vaccine through the controlled vaccination. $^{3-5)}$ This peptide-vaccine therapy is expected to lead the regression of disease-related cells without damaging normal tissues. Since the combination of HLA genes has a large diversity, the selection of peptide adequate for each individual is one of the important issues to enhance the performance of immunotherapy.
The discovery of a peptide inducing strong immunological response is a key factor in immunotherapy. Since the peptide firmly bound to HLA molecule will be effective as medical material, the search of potent peptide, usually called as epitope, is critically important. In this study, we focus on Wilms' tumor (WT1) protein. ${ }^{6)}$ WT1 protein is encoded in $\beta 2$ Wilms' tumor gene and overexpressed in leukemic and solid tumor cell. A 9-mer amino acid peptide encoded in WT1 protein was already known to work as an antigenic peptide for HLA-A*2402 molecule. Oka and his co-workers reported the experimental findings that the replacement of the second amino acid residue, which is considered to be deeply responsible for the peptide binding to HLA-A*2402, induced strong immunological response of WT1 specific CTLs compared to the natural peptide. A peptide containing a single amino residue mutation is currently applies for the clinical trial for a vaccination against solid tumor or leukemia. ${ }^{7)}$

Computer simulation enables us to visualize the interaction of proteins or the interaction of a chemical compound and its target protein. Molecular dynamics (MD) is one of the major computational approaches for analyzing the interaction between epitope peptide and HLA molecules to predict a suitable epitope inducing immunological response. The feasibility of MD simulation was examined through a comparison with experimental methods. ${ }^{8,9)}$ In this work, we employed MD simulation to analyze the interaction between HLA molecule and epitope peptide. Atoms in the molecules are described as charged particles, and motions of the respective particles can be predicted in a time-evolution manner with calculating the forces among charged particles. Hence MD simulation produces a virtual system in which physiological conditions such as temperature and pressure are set equal to the designated experimental setup.

We also performed computational analysis for estimating 
the binding affinity between epitope peptide and HLA- $\beta 2 \mathrm{~m}$ heterodimer. Several computer programs to predict the binding affinity of peptides to HLA were already released so far. MHCpred ${ }^{10)}$ and PepDict ${ }^{11)}$ are well known programs for HLA-peptide prediction. Those programs were developed on the knowledge-based approach or the neural network method making use of in vitro experimental data. These programs effectively and accurately predict the peptide binding with respect to the known kinds of HLAs. However, the accuracy of the prediction is insufficient for variety kinds of HLAs, especially for the HLA of which little experimental data are available. In this study, we carried out molecular dynamics simulation, followed by molecular mechanics generalized Born/surface area (MM-GB/SA) method and normal mode analysis. ${ }^{12)} \mathrm{MD}$ simulation provides a stable molecular structure of a complex of HLA- $\beta 2 \mathrm{~m}$ heterodimer and epitope peptide. MM-GB/SA method and normal mode analysis gives the binding free energy of the peptide to HLA, which directly corresponds to the binding affinity. The present computation is not knowledge-based approach and then requires no experimental data. Hence, the calculation method employed in this study will be applied to not only to major HLAs but also rare HLA molecules.

In the first step of this study, we built calculation models for a complex of HLA-A*2402 and WT1-derived epitope peptide; CMTWNQMNL, and also a complex of HLAA*2402 and every kind of single amino residue-mutated peptides. MD simulation was applied to these complex models to analyze the binding structure and to estimate the binding free energy. Simulation showed a difference in binding free energy between the natural peptide and the mutated ones. The discussion was developed in terms of compatibility with the experimentally measured immunological response. Since the second residue at N-terminus of epitope (p2) is important for the peptide binding, the interactions of $\mathrm{p} 2$ residue with amino residues in HLA molecule were closely investigated. This investigation will be helpful for designing the epitope peptide enhancing the binding to HLA molecules.

\section{Experimental}

Build of Simulation Model A structure of HLA-A*2402 was already resolved by an $\mathrm{X}$-ray crystallographic analysis and registered in protein data bank $(\mathrm{PDB})^{13)}$ in a complex form with an antigen peptide; PDB code $2 \mathrm{BCK}$, in a resolution of $2.80 \AA{ }^{14)}$ This crystal structure contains two pairs of HLA$\beta 2 \mathrm{~m}$ complexed with epitope peptides, sulfate ions, glycerols, and waters, while there are 8 missing residues. The epitope peptide in 2BCK structure is a 9-mer fragment of telomerase reverse transcriptase and the sequence is different from WT1 protein. Then, computational model was built by homology modeling. One of the HLA- $\beta 2 \mathrm{~m}$-epitope complexes in $2 \mathrm{BCK}$ crystal structure was selected as a reference. Modeller9.2 was employed for homology modeling, ${ }^{15-17)}$ in which the original epitope peptide in the crystal structure was replaced by WT1 peptide. Further, p2 residue was converting from methionine of the natural peptide into other kinds of amino acids. The complex was solvated with about 5000 TIP3P waters, ${ }^{18)}$ generated in a rectangular box using leap module of AMBER $9^{19,20)}$ in a manner shown in Fig. 1 a.

Computational Procedure MD simulation was carried out using sander module of AMBER9 program package. ${ }^{20)}$ AMBER ff03 force field was employed. MD simulation was executed in three steps of minimization, heating and equilibration. Atom geometry was energetically minimized in the first step. The minimization was performed with the steepest descent method $^{21)}$ for the earlier 3000 cycles and with the conjugated gradient method for the later 10000 cycles, with only water molecules allowed to move freely. The minimization was executed in a similar manner, with water molecules and epitope peptides allowed to move. Again the minimization was executed without any constraint for waters, peptide and HLA- $\beta 2 \mathrm{~m}$. The heating calculation was executed in the NVT ensemble condition. The temperature of the model system was elevated from 0 to $310 \mathrm{~K}$ for $200 \mathrm{ps}$. The equilibration calculation was performed in the NPT ensemble condition with a temperature of $310 \mathrm{~K}$ and a pressure of $1 \mathrm{~atm}$. Langevin dynamics method was applied for controlling temperature and pressure with a collision frequency of $0.1 \mathrm{ps}^{-1}$. $^{22-24)}$ The long-distance electrostatic and van der Waals interaction was calculated under the periodic boundary condition, setting the cutoff distance to be $12 \AA$. The expansion and shrinkage of all covalent bonds connecting to hydrogen atoms were constraint with SHAKE algorithm. ${ }^{25)}$ The integration time step was $2 \mathrm{fs}$. Six nanoseconds equilibration calculation was carried out for all models.

Estimation of Binding Affinity The binding affinity of HLA-A*2402$\beta 2 \mathrm{~m}$ and epitope peptide was estimated from the binding free energy $\Delta G_{\text {bind }}$, ${ }^{26)}$

$$
\Delta G_{\text {bind }}=\Delta H_{\text {bind }}-T \Delta S_{\text {bind }}
$$

in which the enthalpic and entropic terms were computed by MM-GB/SA $\operatorname{method}^{27)}$ and normal mode analysis, ${ }^{12)}$ respectively.

Five hundred snapshot structures were extracted from the trajectory of the last $1 \mathrm{~ns}$ run of the equilibrating MD simulation. For every snapshot structure, binding enthalpy was calculated by MM-GB/SA method and the average value of the binding enthalpy was obtained. Ten snapshot structures were extracted from the last $1 \mathrm{~ns}$ trajectory of MD simulation to calculate entropic term with the normal mode analysis and the averaged value was obtained for the entropic term. In the calculation of enthalpic term, the respective model structures for HLA- $\beta 2 \mathrm{~m}$, epitope peptide, and HLA- $\beta 2 \mathrm{~m}$-peptide complex were built from each of 500 snapshot structures, and $\Delta H_{\text {bind }}$ was computed with the following equation.

$$
\Delta H_{\text {bind }}=H_{\text {complex }}-\left(H_{\text {HLA- } 32 \mathrm{~m}}+H_{\text {peptide }}\right)
$$

where $\Delta H_{\mathrm{HLA}-\beta 2 \mathrm{~m}}, \Delta H_{\text {peptide }}$, and $\Delta H_{\text {complex }}$ represent the free energies of HLA- $\beta 2 \mathrm{~m}$, epitope peptide and their complex, respectively. In the normal mode analysis, the entropy for rotation and vibration was computed using the models for HLA- $\beta 2 \mathrm{~m}$, epitope peptide, and their complex and the change in entropy due to peptide binding was obtained from the following equation.

$$
T \Delta S_{\text {bind }}=T S_{\text {complex }}-\left(T S_{\text {HLA- } \beta 2 \mathrm{~m}}+T S_{\text {peptide }}\right)
$$

In this study, we introduced the deformation effect of epitope peptides due to binding to HLA. The ligand molecule in this work is a 9-mer peptide chain. The peptide chain has a large conformational diversity due to the rotation around the covalent bond. That is, 9-mer peptide shows a considerably large difference in structure between the HLA-bound and unbound states. This is a marked difference from low mass-weight compounds like medical drugs. In the usual MM-GB/SA method, the structure of ligand is assumed to be identical between the receptor-bound and unbound states. However the estimation of enthalpic and entropic component is not accurate if the diversity in configuration of the ligand molecule is large. The deformation effect should be taken into account to improve the energy estimation. In order to incorporate the deformation effect, additional MD simulations were performed for the model representing epitope peptide only, followed by evaluation of the binding free energy with MM-GB/SA method and normal mode analysis. The epitope peptide was placed in a rectangular box and solvated with about 5000 water molecules. The cutoff distance for electrostatic and van der Waals forces was set to $12 \AA$. Totally $6 \mathrm{~ns}$ MD simulation was performed for equilibration and the trajectory for the last $1 \mathrm{~ns}$ was used for estimating the binding energy. Snapshot structures were extracted every $10 \mathrm{ps}$ from the last $1 \mathrm{~ns}$ MD simulation and 100 structures were collected. MMGB/SA method and normal mode analysis were executed to compute the enthalpic and entropic contributions. The modified binding free energy was obtained from the following equations,

$$
\begin{aligned}
& \Delta G_{\text {mod }}=\Delta H_{\text {mod }}-T \Delta S_{\text {mod }} \\
& \Delta H_{\text {mod }}=H_{\text {complex }}-\left(H_{\text {HLA }}+H_{\text {peptide }}^{\prime}\right) \\
& T \Delta S_{\text {mod }}=T S_{\text {complex }}-\left(T S_{\text {HLA }}+T S_{\text {peptide }}^{\prime}\right)
\end{aligned}
$$

where $H_{\text {peptide }}^{\prime}$ and $T S_{\text {peptide }}^{\prime}$ were calculated from MD simulation for epitope peptide only.

In order to examine the interaction between the side chain of $\mathrm{p} 2$ residue of epitope peptide and all the residues of HLA molecule, alanine-scanning calculation $^{28,29)}$ was performed, in which alanine was substituted for the amino acid at the residue of which the interaction energy with $\mathrm{p} 2$ residue was estimated. Comparison in energy between substituted and non-substituted mod- 
els indicated the contribution of the residue to the total binding energy. The binding free energies between $\mathrm{p} 2$ residue and the residues of HLA were calculated by MM-GB/SA method. ${ }^{27)}$

\section{Results and Discussion}

Binding Structure of Epitope Peptide to HLA Class I Molecule HLA class I molecule has six binding pockets labeled $\mathrm{A}-\mathrm{F}$ as shown in Fig. 1b. The binding of epitope peptide to the pockets will stabilize the complex structure. ${ }^{14,30)}$ The amino acid residue of the peptide playing an important role in binding to HLA- $\beta 2 \mathrm{~m}$ is called "anchor residue." The position of anchor residue and the location of significantly important pocket vary depending on the type of HLA. The second residue from N-terminus; $\mathrm{p} 2$, and the ninth residue; p9, are the anchor residues for HLA-A*2402. The favorable amino acid for anchor residue is also changed, depending on the type of HLA. For example Tyr or Phe at p2 residue makes the peptide binding strong in HLA-A*2402, ${ }^{14)}$ while Leu or Met generates a firm binding to HLA-A*2402. ${ }^{31)}$. According to the experiment by Tsuboi et al., ${ }^{7)}$ the substitution of Tyr for Met at p2 anchor residue makes the binding of WT1 peptide stronger. The residues responsible for the peptide binding to HLA are supposed to have little influence on the association with CTL. Therefore, the replacement of anchor residue with other kind of amino acids will be one of the effective approaches to enhance the binding of epitope peptide to HLA. In this study, the binding affinity was examined with changing the amino acid at $\mathrm{p} 2$ anchor residue of the natural WT1 peptide.

Snapshot structures for analyzing binding mode and binding affinity were extracted from the last $1 \mathrm{~ns}$ trajectory of MD simulation. The model system should be sufficiently equilibrated for the sake of reliable analysis. One of the standard techniques to confirm the equilibration is to measure the root mean square deviation (RMSD) value between the snapshot structure at the time point concerned and the structure at the starting point. If the time evolution of RMSD shows constant, the system fluctuates in a single conformation and is confirmed to be equilibrated. RMSD value during MD simulation was monitored on the HLA- $\beta 2 \mathrm{~m}$ peptide complex, with respect to the $\mathrm{C} \alpha, \mathrm{N}$, and $\mathrm{C}$ main chain atoms, setting the snapshot structure after heating calculation as a reference at the starting point. The RMSD plot indicates that RMSD value fluctuates in a range of $2.8 \pm 0.5 \AA$ for the last $1 \mathrm{~ns}$ (data not shown). This result means that the model system was in equilibration.

Principal component analysis ${ }^{32,33)}$ is another method to confirm the equilibration of the calculation system. Since protein usually fluctuates and has several probable confirmations, there exist several minimum points in potential energy surface. If the energy barrier separating potential minima is low enough, the structure fluctuates among some energetically minimized points during MD simulation. When the model system is in equilibration, the structure fluctuates only around a stable minimum point. Principle component analysis was executed for the calculations on the natural peptide and 19 mutants (data not shown). In every case, there appear several clusters, and the conformation converges to a single cluster with the progress of MD simulation. This result suggests that $6 \mathrm{~ns} \mathrm{MD}$ simulation leads structural equilibration for the HLA- $\beta 2$ m-peptide complex.

Binding Free Energy Table 1 shows the comparison of the calculated binding free energy, and the binding constant obtained from experiment for HLA-A*2402 bound with the natural peptide and $\mathrm{M} 2 \mathrm{Y}$ variant. The binding constant $K_{\mathrm{d}}$ in Table 1 was measured in the in vitro experiment using flow cytometry by Oka et al. ${ }^{7)}$ According to their in vitro experiment, $K_{\mathrm{d}}$ value for the natural peptide was measured to be $1.82 \times 10^{-5} \mathrm{M}$ and that for M2Y variant was $6.40 \times 10^{-7} \mathrm{M}$. Hence, M2Y showed a higher binding affinity to HLA-

(a)
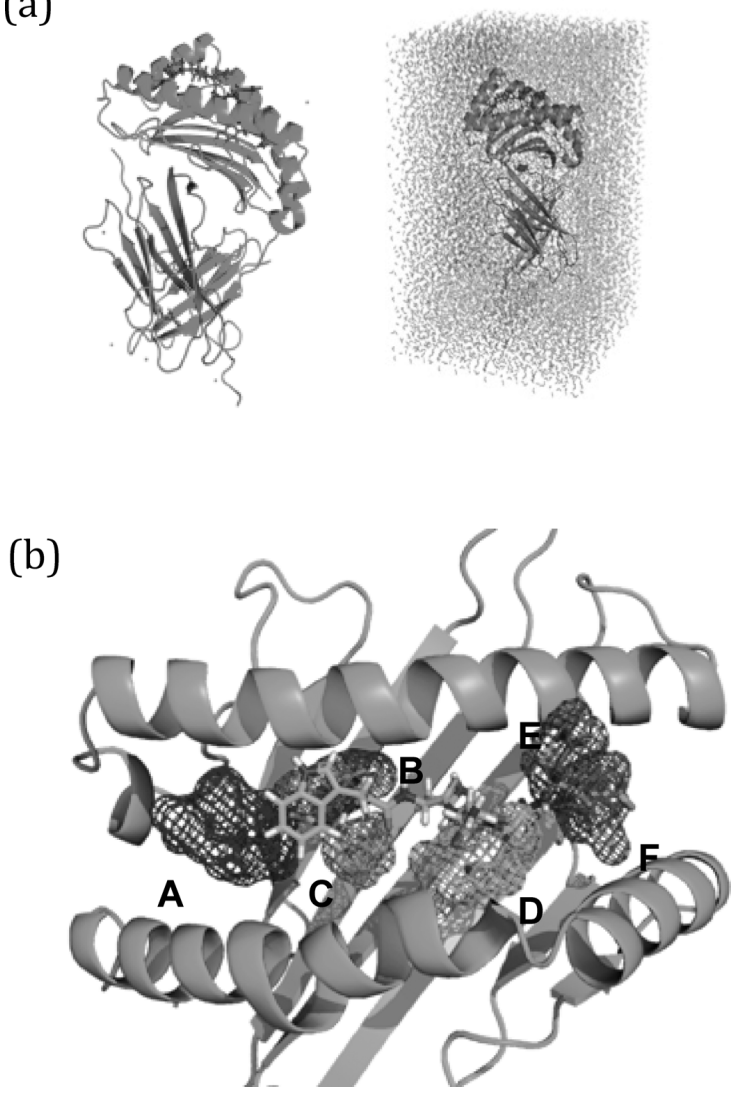

Fig. 1. (a) Structure of HLA Class I Molecule Combined with 9-mer Peptide

9-mer peptide is shown in the stick representation. 9-mer peptide and HLA complex was solvated with about 5000 of TIP3P waters, generated in a rectangular box for molecular dynamics simulation.

(b) Structure of Binding Pockets of HLA Class I Molecule

This molecule has six binding pockets labeled A-F. The binding of an epitope peptide into the pockets will stabilize the complex.

Table 1. Comparison of the Calculated Binding Free Energy and the Experimentally Measured Binding Constant

\begin{tabular}{|c|c|c|c|c|c|}
\hline Peptide & A sequence & $K_{\mathrm{d}}(\mathrm{M})$ & $\Delta G_{\exp }(\mathrm{kcal} / \mathrm{mol})$ & $\Delta G_{\text {bind }}(\mathrm{kcal} / \mathrm{mol})$ & $\Delta G_{\text {mod }}(\mathrm{kcal} / \mathrm{mol})$ \\
\hline NP & CMTWNQMNL & $1.82 \times 10^{-5}$ & & -59.6 & -44.8 \\
\hline M2Y & CYYTWNQMNL & $6.40 \times 10^{-7}$ & -2.0 & -64.4 & -45.7 \\
\hline
\end{tabular}


Table 2. Calculated Binding Free Energy for Every Kind of Peptides Combined with HLA

\begin{tabular}{|c|c|c|c|c|c|c|c|}
\hline Peptide & $\begin{array}{c}\Delta H_{\mathrm{ele}} \\
(\mathrm{kcal} / \mathrm{mol})\end{array}$ & $\begin{array}{c}\Delta H_{\mathrm{vwd}} \\
(\mathrm{kcal} / \mathrm{mol})\end{array}$ & $\begin{array}{c}\Delta H_{\mathrm{int}} \\
(\mathrm{kcal} / \mathrm{mol})\end{array}$ & $\begin{array}{c}\Delta H_{\text {sol }} \\
(\mathrm{kcal} / \mathrm{mol})\end{array}$ & $\begin{array}{c}\Delta H_{\text {mod }} \\
(\mathrm{kcal} / \mathrm{mol})\end{array}$ & $\begin{array}{c}T \Delta S_{\text {mod }} \\
(\mathrm{kcal} / \mathrm{mol})\end{array}$ & $\begin{array}{c}\Delta G_{\mathrm{mod}} \\
(\mathrm{kcal} / \mathrm{mol})\end{array}$ \\
\hline NP & -139.8 & -89.0 & 6.4 & 144.2 & -78.2 & -33.4 & -44.8 \\
\hline $\mathrm{M} 2 \mathrm{Y}$ & -159.5 & -93.4 & 10.0 & 157.4 & -85.5 & -39.8 & -45.7 \\
\hline M2W & -190.8 & -93.7 & 10.6 & 188.2 & -85.7 & -37.7 & -48.2 \\
\hline $\mathrm{M} 2 \mathrm{~V}$ & -153.0 & -71.1 & 5.5 & 152.4 & -66.2 & -37.7 & -28.5 \\
\hline M2I & -155.0 & -75.0 & 5.1 & 152.4 & -72.5 & -32.2 & -40.3 \\
\hline M2L & -148.6 & -77.3 & 0.4 & 150.5 & -75.0 & -46.1 & -28.9 \\
\hline $\mathrm{M} 2 \mathrm{~A}$ & -142.1 & -71.2 & 2.4 & 147.0 & -63.9 & -31.6 & -32.3 \\
\hline $\mathrm{M} 2 \mathrm{~F}$ & -101.3 & -71.1 & 1.8 & 106.4 & 64.2 & -39.2 & -25.0 \\
\hline $\mathrm{M} 2 \mathrm{G}$ & -152.9 & -80.3 & 7.0 & 153.9 & -72.3 & -38.0 & -34.3 \\
\hline M2S & -173.7 & -74.9 & 2.2 & 167.4 & -79.0 & -37.5 & -41.5 \\
\hline M2T & -177.0 & -73.5 & 4.6 & 176.4 & -69.5 & -30.6 & -38.9 \\
\hline $\mathrm{M} 2 \mathrm{P}$ & -182.2 & -84.7 & 8.1 & 180.1 & -78.7 & -37.4 & -41.3 \\
\hline $\mathrm{M} 2 \mathrm{C}$ & -185.0 & -89.4 & 6.7 & 183.5 & -84.2 & -40.8 & -43.5 \\
\hline $\mathrm{M} 2 \mathrm{~N}$ & -163.4 & -84.6 & 6.1 & 165.2 & -76.7 & -43.4 & -33.3 \\
\hline M2Q & -187.2 & -83.7 & 6.0 & 182.7 & -82.2 & -38.7 & -43.5 \\
\hline $\mathrm{M} 2 \mathrm{H}$ & -157.8 & -83.5 & 5.4 & 161.3 & -74.6 & -33.9 & -40.7 \\
\hline $\mathrm{M} 2 \mathrm{~K}$ & -234.3 & -65.9 & 3.5 & 239.1 & -57.6 & -39.0 & -18.6 \\
\hline M2R & -257.7 & -83.6 & 5.6 & 261.3 & -74.3 & -45.0 & -29.3 \\
\hline M2D & -102.9 & -79.9 & -0.8 & 110.0 & -73.6 & -44.9 & -28.7 \\
\hline M2E & -63.3 & -77.6 & 5.0 & 71.5 & -64.1 & -32.4 & -31.7 \\
\hline
\end{tabular}

NP, natural peptide; $\Delta H_{\text {ele }}$, electrostatic energy; $\Delta H_{\text {vdw }}$, van der Walls interaction; $\Delta H_{\text {int }}$, internal energy from bond, angle and dihedral; $\Delta H_{\text {sol }}$, solvation energy; $\Delta H_{\text {mod }}$, modified averaging enthalpic energy; $T \Delta S_{\text {mod }}$, modified averaging entopic energy; $\Delta G_{\text {mod }}$, modified binding free energy.

A*2402, compared to the natural peptide. The calculation of binding free energy without deformation effect, $\Delta G_{\text {bind }}$, indicated that M2Y mutant is more stable than the natural peptide. Accordingly, the usual technique for calculating binding free energy could predict the binding affinity measured in experiments. $\Delta G_{\text {mod }}$ of Table 1 shows a comparison in energy between the natural peptide and $\mathrm{M} 2 \mathrm{Y}$ variant obtained with the deformation effect of epitope peptide took into account. The energy comparison shows that the binding of M2Y peptide is more stable than the natural peptide, which is also compatible with the experimental findings. The energy difference estimated from the binding constant is $-2.0 \mathrm{kcal} / \mathrm{mol}$ between the natural peptide and M2Y. The calculated energy difference is $-0.9 \mathrm{kcal} / \mathrm{mol}$. Accordingly the combination of MM-GB/SA method and normal mode analysis with considering the deformation effect will correctly predict the binding affinity of epitope peptide bound to HLA- $\beta 2 \mathrm{~m}$. The result suggests that the deformation effect has a certain degree of importance in the prediction of the binding free energy.

It is informative to clarify what chemical structure increases the binding affinity between HLA- $\beta 2 \mathrm{~m}$ and epitope peptide. Hence, the $\mathrm{p} 2$ amino residue of the peptide was replaced with another kind of amino acids. The complex models for 19 kinds of variants bound to HLA- $\beta 2 \mathrm{~m}$ were built and MD simulations were performed for all models, followed by free energy calculation with MM-GB/SA method and normal mode analysis. Our concern is how we can improve the binding affinity replacing an amino acid with another one to increase the electrostatic or van der Waals interaction. Tyr and Trp have aromatic ring and contain polar atoms possible for hydrogen bonding. Non-polar amino acid residues, Val, Leu, Ile, Phe, Pro, were substituted at p2 anchor position, because they have large hydrophobic side chain to increase van der Waals interaction. Ser and Thr have hydroxy group in their side chains to make it possible to form hydrogen bonds. Cys, Asn and Gln can also make hydrogen bonds because of the presence of polar hydrogen atoms in their side chains.
Basic polar amino acid residues, His, Lys, Arg, and acidic polar amino acid ones, Asp, Glu, can generate ionic interaction while hydrophobic interaction is little. Non-polar amino acid residues, Gly, Ala, have small side chains, but may contribute to keep peptide flexibility.

Table 2 shows the calculated binding free energy for the natural and other 19 kinds of peptide complexed with HLAA*2402 molecules. The energy difference in $\Delta G_{\text {mod }}$ indicates that all of substitutions except for $\mathrm{M} 2 \mathrm{Y}$ and $\mathrm{M} 2 \mathrm{~W}$ are less stable than the natural peptide. $\Delta H_{\text {mod }}$ in the table also indicates that HLA- $\beta 2 \mathrm{~m}$ complexed with M2Y or M2W mutant show the lowest energy among all the complexes. Namely these epitope peptides will give the highest binding affinity. It is already known that a modified 9-mer WT1 (M2Y) peptide showed much higher HLA-A*2402-binding affinity than the natural 9-mer WT1 peptide. ${ }^{7)}$ In this study, we found that the binding free energy of M $2 \mathrm{~W}$ to HLA- $\beta 2 \mathrm{~m}$ is slightly lower than M2Y $(-2.5 \mathrm{kcal} / \mathrm{mol})$. This means that $\mathrm{M} 2 \mathrm{~W}$ peptide substitution is the most favorable in terms of binding affinity. The van der Waals energy $\left(\Delta H_{\mathrm{vdw}}\right)$ shows a favorable contribution to the peptide binding. $\Delta H_{\mathrm{vdw}}$ value of the HLA$\beta 2 \mathrm{~m}$ complexed with $\mathrm{M} 2 \mathrm{Y}$ or $\mathrm{M} 2 \mathrm{~W}$ is the lowest among the complexes. The van der Waals energy of M2W is slightly lower then M2Y $(-0.03 \mathrm{kcal} / \mathrm{mol})$. Therefore M2W peptide substitution is more favorable because of the presence of large hydrophobic side chain. Among the other peptides M2K shows the highest $\Delta H_{\mathrm{vdw}}$ value and highest binding free energy $\Delta G_{\text {mod }}$, which suggests that the presence of Lys causes a strong instability for the binding of HLA- $\beta 2 \mathrm{~m}$ and peptide. The large change in $\Delta G_{\bmod }$ in Table 2 clearly demonstrates that the side chain of the anchor residue, $\mathrm{p} 2$, plays a quite important role for peptide binding. M2C, M2Q, $\mathrm{M} 2 \mathrm{~S}$ and M2P show the binding energies near to the natural peptide, which suggests that side chain hydrogen-bond doner or acceptor can serve as an anchor for peptide binding. Judging from the binding energy, M2H, M2I, M2T, M2G, M2N, $\mathrm{M} 2 \mathrm{~A}$ and M2E are not so stable. Further, M2V, M2F, M2L, 
NP

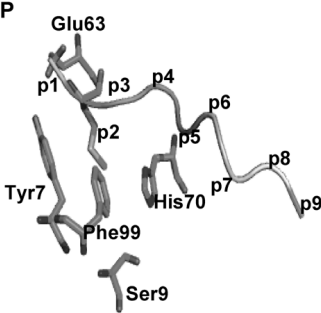

M2V

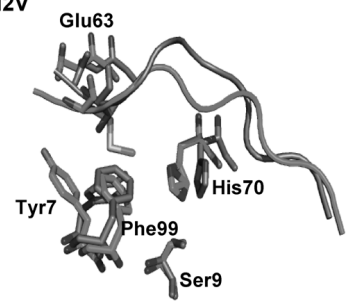

M2Y

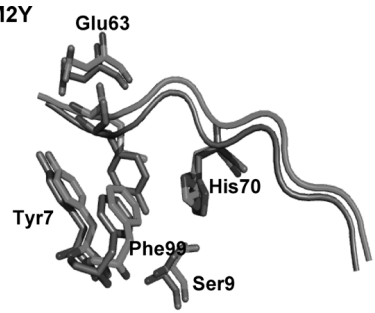

M2F

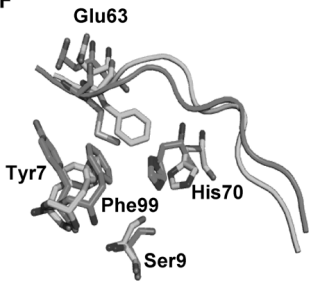

M2W

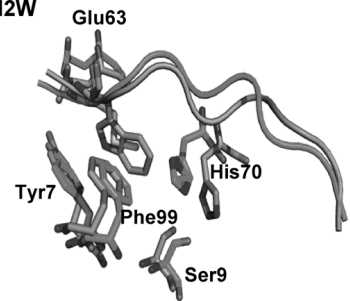

M2G

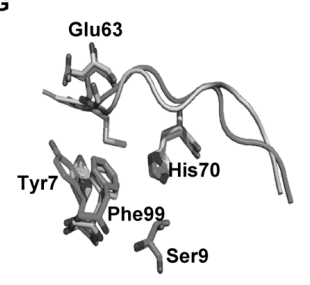

M2S

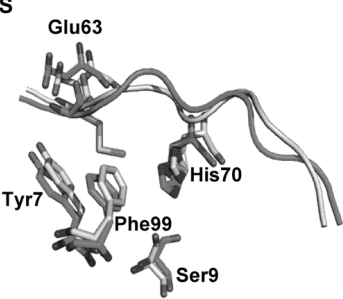

M2Q

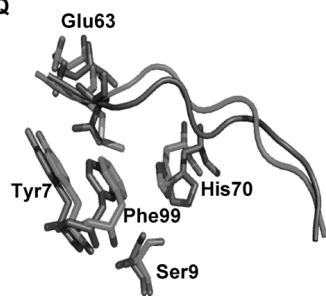

M2T

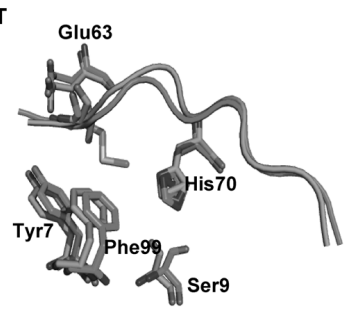

M2R

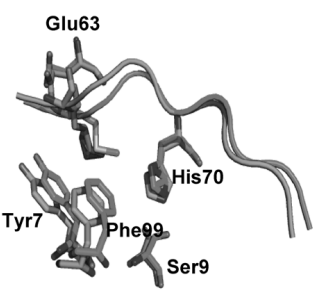

M2N

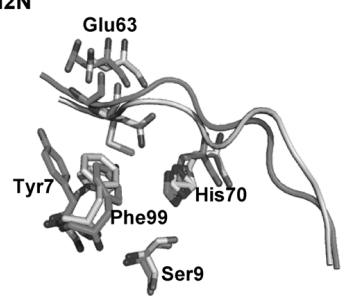

M2D

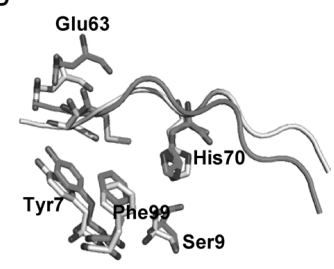

Fig. 2. Superimposition of the Average Structure of 19 Kinds of Peptide Variants on That of the Natural Peptide Bound to HLA- $\beta 2 \mathrm{~m}$

The structure of the natural peptide complexed with HLA- $\beta 2 \mathrm{~m}$ is colored dark in superimposition. The position of each residue on the epitope peptide is shown in the figure at the right bottom for natural peptide (NP).

M2D and M2R hardly maintain the stability of peptide binding.

Binding Structure Figure 2 shows the superimposition of the averaged structures of 19 kinds of peptide to the natural peptide bound to HLA- $\beta 2 \mathrm{~m}$. The averaged structure for the last $1 \mathrm{~ns}$ MD simulation was obtained for the respective models, and pymol software ${ }^{35)}$ was employed to superimpose the structure of the HLA- $\beta 2 \mathrm{~m}$-natural peptide complex on that of the $\mathrm{p} 2$-mutated one, with respect to main chain atoms of HLA. The N-terminal sides of the peptide main chain for M2F and M2D models are largely deviated from that of the natural peptide. These peptides showed the instability in binding to HLA- $\beta 2 \mathrm{~m}$ molecule in Table 2 . The C-terminal side of the epitope peptide is largely deviated in M2D, M2N and $\mathrm{M} 2 \mathrm{G}$ models from the natural peptide. This indicated that the C-terminal region of M2D, M2N and M2G peptides is loosely bound to HLA. Several X-ray crystal structures obtained for other types of HLA indicated that no significant deviation was observed for the three residues at the both ter- minal regions. ${ }^{36)}$ This suggests that central residues of the epitope peptide are mainly responsible for the activation of CTL and the terminal residues primarily contribute to the binding of epitope peptide to HLA. Both terminal regions of M2Y, M2W, M2Q and M2S are compatible with that of the natural peptide. All these peptide show the low binding energy in Table 2 and the complexes with HLA are stable.

Contribution of Amino Residues of HLA to Peptide Binding The interaction energy between $\mathrm{p} 2$ anchor residue and the respective residues of HLA were calculated by alanine scanning technique. ${ }^{28,29)}$ In the alanine scanning calculation, one of the residues of HLA is replaced by Ala and then the binding energy is calculated with the residue-replaced model. A comparison in binding free energy between the models with and without replacement indicates the contribution of the residue to the peptide binding. The energy contribution of $\mathrm{p} 2$ residue is computed by MM-GB/SA method. Figure 3 shows the results of alanine scanning calculation, in which low energy value means positive contribution of the 

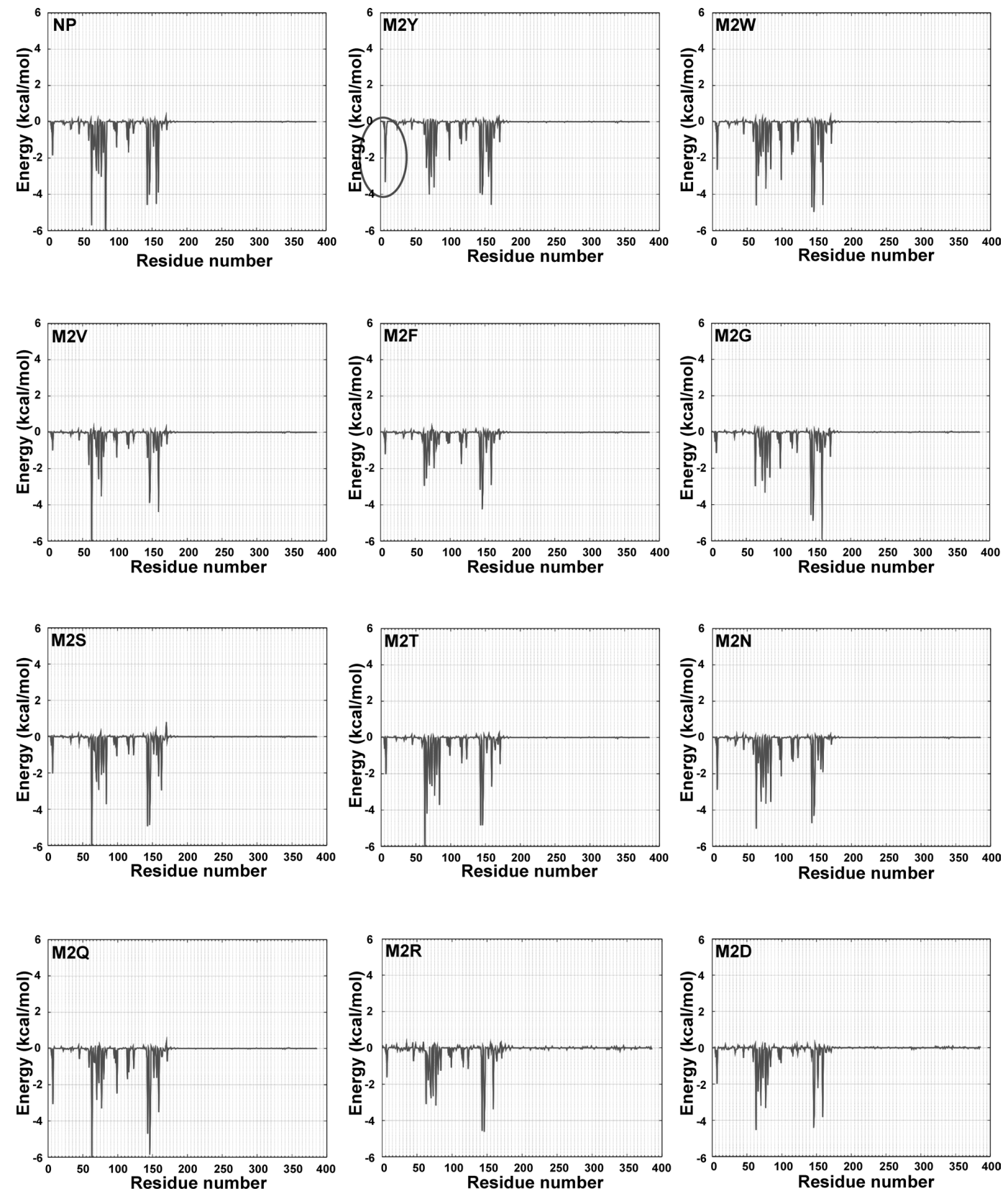

Fig. 3. Interaction Energy of p2 Residue of Epitope Peptide with the Respective Residues of HLA Class I Molecule

A comparison of the energy diagrams suggests that several residues concerning the interaction are common among the mutants but the interaction with Ser9 residue of HLA is only enhanced in M2Y.

residue to the interaction with $\mathrm{p} 2$ anchor residue. In all HLA$\beta 2 \mathrm{~m}$-peptide models, Tyr7, Glu63, His70, Phe99, Ser9 are related to the interaction. The locations of these residues are displayed in Fig. 3 to clarify the relationship with p2 anchor residue. These residues were reported to be located at the deep bottom of B-pocket. ${ }^{30)}$ A comparison in Fig. 3 suggests that the interaction with Ser9 of HLA residue is markedly enhanced in M2Y (gray circle in Fig. 3). M2Y generates a hydrogen bond with HLA, which makes the peptide binding stable. The interaction with Ser9 of HLA residue is also enhanced in $\mathrm{M} 2 \mathrm{~W}$. The time ratio for keeping hydrogen bond within $3.5 \AA$ was calculated from the last $1 \mathrm{~ns}$ MD simulation. Ser9 keeps a hydrogen bond to M2Y for $74 \%$ of MD simulation time, while other models scarcely keep the hydrogen bond with Ser9. The interaction with Phe99 is the high- est in M2W. This large interaction will be attributed to the $\pi-\pi$ interaction between the indole group of Trp at $\mathrm{p} 2$ anchor residue and the aromatic ring of Phe. As for M2Y, M2Q and M2N, the interaction with Phe99 is also higher compared with the natural peptide. In M2Q, M2S, M2T and M2V, the interaction with Glu63 was increased while other peptides scarcely showed the favorable energy gain in the interaction with Glu63. In M2Q, M2S, M2T and M2V, several residues of protein also show small degree of interaction with $\mathrm{p} 2$ residue.

Table 3 shows the respective components of the binding free energy between $\mathrm{p} 2$ residue and HLA- $\beta 2 \mathrm{~m}$, where $\Delta H_{\text {ele-p2 }}, \Delta H_{\text {vdw-p2 }}$, and $\Delta H_{\text {sol-p2 }}$ represent the electrostatic, van der Waals, and solvation energies, respectively. There are many hydrophobic residues at the inside of B binding pocket 
Table 3. Components of the Binding Free Energy between p2 Residue and HLA

\begin{tabular}{lrrrrr}
\hline \hline Peptide & $\begin{array}{c}\Delta H_{\text {ele-p2 }} \\
(\mathrm{kcal} / \mathrm{mol})\end{array}$ & $\begin{array}{c}\Delta H_{\mathrm{vwd}-\mathrm{p} 2} \\
(\mathrm{kcal} / \mathrm{mol})\end{array}$ & $\begin{array}{c}\Delta H_{\text {int-p2 }} \\
(\mathrm{kccal} / \mathrm{mol})\end{array}$ & $\begin{array}{c}\Delta H_{\text {sol-p2 }} \\
(\mathrm{kcal} / \mathrm{mol})\end{array}$ & $\begin{array}{c}\Delta H_{\mathrm{p} 2}{ }^{*} \\
(\mathrm{kcal} / \mathrm{mol})\end{array}$ \\
\hline NP & -20.7 & -10.1 & 8.1 & 5.3 & -17.4 \\
M2Y & -17.6 & -13.4 & 7.9 & 4.6 & -18.5 \\
M2W & -17.8 & -15.4 & 8.6 & 7.0 & -17.6 \\
M2V & -27.4 & -6.8 & 8.2 & 3.3 & -22.7 \\
M2I & -23.8 & -8.7 & 8.1 & 4.9 & -19.9 \\
M2L & -19.5 & -7.9 & 7.8 & 2.8 & -16.8 \\
M2A & -20.3 & -4.9 & 8.1 & 3.5 & -13.6 \\
M2F & -15.9 & -9.5 & 8.5 & 1.0 & -15.9 \\
M2G & -12.6 & -3.6 & 9.2 & 2.0 & -5.0 \\
M2S & -39.5 & -3.0 & 7.7 & 14.7 & -20.1 \\
M2T & -19.6 & -6.9 & 7.7 & 6.7 & -12.1 \\
M2P & -9.9 & -7.7 & 9.3 & 1.9 & -6.4 \\
M2C & -20.7 & -6.0 & 7.8 & 4.5 & -14.4 \\
M2N & -23.8 & -8.2 & 8.6 & 9.6 & -13.8 \\
M2Q & -30.7 & -8.8 & 8.2 & 14.1 & -17.2 \\
M2H & -21.3 & -10.3 & 8.0 & 7.5 & -16.1 \\
M2K & -115.4 & -6.9 & 8.1 & 104.0 & -10.2 \\
M2R & -103.7 & -10.5 & 8.5 & 93.8 & -11.9 \\
M2D & 25.5 & -6.8 & 7.6 & -36.6 & -10.3 \\
M2E & 31.7 & -7.3 & 8.7 & -44.0 & -10.9 \\
\hline
\end{tabular}

NP, natural peptide; $\Delta H_{\text {ele-p2 }}$, electrostatic energy; $\Delta H_{\text {vdw-p2 }}$, van der Walls interaction; $\Delta H_{\text {int-p2 }}$, internal energy from bond, angle and dihedral; $\Delta H_{\text {sol-p2 }}$, solvation energy; $\Delta H_{\mathrm{p} 2}{ }^{*}$, total binding free energy between $\mathrm{p} 2$ residue and HLA- $\beta 2 \mathrm{~m}$.

of HLA. This suggests that hydrophobic residues of epitope peptide play an important role in peptide binding. For example, $\Delta H_{\mathrm{vdw}-\mathrm{p} 2}$ of M2I, M2L and M2F are slightly larger than those of other mutations into a similar size of residue and further $\Delta H_{\text {sol-p2 }}$ values of these residues are markedly low. Then, M2I, M2L and M2F indicate the favorable total binding energy in $\Delta H_{\mathrm{p} 2}$ compared with the polar residues of M2K, M2R, M2D, M2E as seen in Table 3. $\Delta H_{\mathrm{vdw}-\mathrm{p} 2}$ of M2S and $\mathrm{M} 2 \mathrm{G}$ are -3.0 and $-3.6 \mathrm{kcal} / \mathrm{mol}$, and they are the highest among 20 peptides. This result is reasonably explained from the smallness of the side chain of Gly and the hydrophilicity of Ser. In contrast, $\Delta H_{\mathrm{vdw}-\mathrm{p} 2}$ of $\mathrm{M} 2 \mathrm{~W}$ is $-15.4 \mathrm{kcal} / \mathrm{mol}$, which is the lowest among the peptides. $\Delta H_{\mathrm{vdw}-\mathrm{p} 2}$ of $\mathrm{M} 2 \mathrm{Y}$ is also lower than other kinds of peptide. The indole group of $\mathrm{M} 2 \mathrm{~W}$ and the aromatic rings of $\mathrm{M} 2 \mathrm{Y}$ peptide have a large contribution to hydrophobic interaction in B-binding pocket. Accordingly, the energetic gain in van der Waals term seems some degree of correlation with the total binding affinity. The p2 anchor residue should have a large hydrophobicity to enhance the binding affinity to HLA$\beta 2 \mathrm{~m}$. The indole group of Trp is important to enhance the binding with the B-pocket of HLA. The hydroxyl group of Tyr is also important for binding with the formation of hydrogen bond. The importance of hydrogen bond by Tyr was also suggested in the recent report that human immunodeficiency virus (HIV) changes its residues from Tyr to Phe to escape from the immunotherapy using a peptide vaccine. ${ }^{37}$

Energy Calculation with Another Type of HLA As an example for predicting binding affinity between epitope and HLA, similar calculation were carried out for HLA-A*0201 and 4 kinds of peptide. ${ }^{36)}$ HLA-A*0201, as well as HLA$A^{*} 2402$, is one of the most popular HLA types in Japan. Table 4 shows the calculated binding free energy, in which computational prediction was compatible with the experimentally measured affinity of the peptide binding to HLA-
Table 4. Calculated Binding Free Energy for Several Peptides Binding to HLA-A*0201

\begin{tabular}{lcrc}
\hline \hline PDB ID & Peptide sequence & $K_{\mathrm{d}}(\mathrm{nm})$ & $\begin{array}{c}\Delta G_{\text {calc }} \\
(\mathrm{kcal} / \mathrm{mol})\end{array}$ \\
\hline $1 \mathrm{HHG}$ & TLTSVNTSV & 294.0 & 6.0 \\
$1 \mathrm{HHJ}$ & ILKEPVHGV & 242.0 & 9.7 \\
$1 \mathrm{HHK}$ & LLFGYPVYV & 11.0 & -1.8 \\
$1 \mathrm{HHI}$ & GILGFVFTL & 6.0 & -30.1 \\
\hline
\end{tabular}

A*0201. These results suggest that our present approach is one of the effective techniques to select the favorable peptides as HLA epitope. We have also examined the role of $\mathrm{p} 2$ residue of peptide in binding to HLA- $\beta 2 \mathrm{~m}$ in this example. In the calculation, a large van der Waals interaction between the side chain of $\mathrm{p} 2$ residue and hydrophobic residues inside B-pocket of HLA was observed.

The application of MD simulation for selecting a peptide strongly bound to HLA was challenged in many research groups. ${ }^{38-41)}$ Although there seems to be a room for improvement in practical use, ${ }^{38)}$ the recent high-performance computers offer us a hope of accurate prediction of antigenic peptides. $^{40)}$ Some theoretical studies suggested the importance of the flexibility for peptide recognition. ${ }^{42,43)} \mathrm{MD}$ simulation is useful to obtain an insight into the dynamic behavior of molecules. For example, an increase in the peptide flexibility was observed in the binding groove. ${ }^{43)}$ The conformational change of HLA molecule due to the peptide binding has been analyzed. ${ }^{4-46)}$ Such a conformational change was shown to be closely related to the recognition of the peptideHLA complex by CTLs, ${ }^{45)}$ and the conformational difference between the peptide-bound and -unbound HLAs was clearly indicated by the removal of the bound peptide from a complex model. ${ }^{46}$ Nojima et al. investigated the structural changes of the peptide-binding groove due to the removal of the peptide using the normal mode analysis. ${ }^{47,48)}$ They suggested that two membrane-proximal domains had significant influence on the activity and stability of HLA. ${ }^{49,50)}$

The computational study is also advantageous to analyze the effect of amino acid substitution of epitope peptides. ${ }^{51-54)}$ The reason for the change in peptide recognition among different types of HLA molecules has been clearly explained. ${ }^{55-57)}$ In the computational studies, the accurate prediction of the complex structure of HLA and peptide is essentially important and some trials were attempted for the construction of appropriate complex structures. ${ }^{58,59)}$ Further, reliable estimation of the binding energy is indispensable for selecting a promising peptide, and MM-GB/SA and MMPoisson-Boltzmann (PB)/SA methods are the most standard approaches for estimating the binding energy. ${ }^{60,61)}$

All the above previous studies suggest the usefulness of molecular simulation for predicting the affinity and specificity of antigenic peptide binding to HLA. Because of the importance of peptide flexibility, the evaluation of entropic terms is indispensable for estimating peptide binding affinity. The prediction of the difference in binding affinity due to the amino acid substitution is possible through the theoretical calculations with high-performance computers of today. Accordingly, our present work will be one of the examples demonstrating an effectiveness of computational analysis, performed by taking full advantage of MD simulation. 


\section{Conclusion}

We investigated the binding affinity between HLAA*2402 and epitope peptide derived from Wilms' tumor (WT1) protein, performing molecular dynamics simulations, followed by MM-GB/SA method and normal mode analysis. The binding affinity was also contrasted between the 9-mer natural WT1 peptide and the modified peptide in which 19 kinds of amino acids were substituted for Met located at the second residue of the N-terminal side. WT1 protein is highly expressed in solid tumor or blood in leukemia patient, and a vaccine targeting WT1 is expected to be one of the promising approaches in immunotherapy. ${ }^{5)}$ In this work, we demonstrated that computational prediction of the peptide binding affinity to HLA is possible. The contribution of $\mathrm{p} 2$ residue of the epitope peptide to the binding free energy was due to the formation of hydrogen bond at the bottom of B-pocket of HLA and van der Waals interaction inside B-pocket. For the stable binding of epitope peptide to HLA-A*2402, p2 residue should have the following property. First, p2 residue should bear alkyl chain or aromatic ring to make van der Waals interaction strong enough inside B-pocket. Second, the residue containing polar atom is advantageous to make a hydrogen bond at the bottom of B-pocket. Third, the residue should have a large volume comparative to Tyr or Phe. These findings will be helpful to design the peptide vaccine inducing high immunological response or the peptide-mimic chemical for medical drugs.

Acknowledgments Calculations were performed at Research Center for Computational Science, Okazaki, Japan, and Information Technology Center of the University of Tokyo, and also by the high-performance computer system at Institute for Media Information Technology of Chiba University. A part of this work was supported by Grant-in-Aid for Scientific Research (C) from Japan Society for the Promotion of Science (JSPS).

\section{References}

1) Rock K. L., Goldberg A. L., Annu. Rev. Immunol., 17, 739-779 (1999).

2) Townsend A., Bodmer H., Annu. Rev. Immunol., 7, 601-624 (1989).

3) Kageyama S., Nagata Y., Miyahara Y., Hiasa A., Naota H., Okumura S., Imai H., Shiraishi T., Masuya M., Nishikawa M., Sunamoto J., Akiyoshi K., Kanematsu T., Scott A. M., Murphy R., Hoffman E. W., Old L. J., Shiku H., Clin. Cancer Res., 12, 7397-7405 (2006).

4) Oka Y., Udaka K., Tsuboi A., Elisseeva O. A., Ogawa H., Aozasa K., Kishimoto T., Sugiyama H., J. Immunol., 164, 1873-1880 (2000).

5) Oka Y., Tsuboi A., Kawakami M., Elisseeva O. A., Nakajima H., Udaka K., Kawase I., Oji Y., Sugiyama H., Curr. Med. Chem., 13, $2345-2352$ (2006).

6) Oka Y., Tsuboi A., Taguchi T., Osaki T., Kyo T., Nakajima H., Elisseeva O. A., Oji Y., Kawakami M., Ikegame K., Hosen N., Yoshihara S., Wu F., Fujiki F., Murakami M., Masuda T., Nishida S., Shirakata T., Nakatsuka S., Sasaki A., Udaka K., Dohy H., Aozasa K., Noguchi S., Kawase I., Sugiyama H., Proc. Natl. Acad. Sci. U.S.A., 101, 1388513890 (2004).

7) Tsuboi A., Oka Y., Udaka K., Murakami M., Masuda T., Nakano A., Nakajima H., Yasukawa M., Hiraki A., Oji Y., Kawakami M., Hosen N., Fujioka T., Wu F., Taniguchi Y., Nishida S., Asada M., Ogawa H., Kawase I., Sugiyama H., Cancer Immunol. Immunother, 51, 614620 (2002).

8) Zhang H., Wang P., Papangelopoulos N., Xu Y., Sette A., Bourne P. E., Lund O., Ponomarenko J., Nielsen M., Peters B., PLoS ONE, 5, e9272 (2010).

9) Morikis D., Lambris J. D., Trends Immunol., 25, 700_707 (2004).

10) Guan P., Doytchinova I., Zygouri C., Flower D., Nucleic Acids Res., 31, 3621-3624 (2003).

11) Tomer Hertz C. Y., BMC Bioinformatics, 7, S3 (2006).

12) Brooks B., Karplus M., Proc. Natl. Acad. Sci. U.S.A., 80, 6571-6575 (1983).
13) Berman H. M., Westbrook J., Feng Z., Gilliland G., Bhat T. N., Weissig H., Shindyalov I. N., Bourne P. E., Nucleic Acids Res., 28, 235242 (2000).

14) Cole D. K., Rizkallah P. J., Gao F., Watson N. I., Boulter J. M., Bell J. I., Sami M., Gao G. F., Jakobsen B. K., Eur. J. Immunol., 36, 170-179 (2006).

15) Sali A., Blundell T. L., J. Mol. Biol., 234, 779-815 (1993).

16) Martí-Renom M. A., Stuart A. C., Fiser A., Sánchez R., Melo F., Sali A., Annu. Rev. Biophys. Biomol. Struct., 29, 291-325 (2000).

17) Fiser A., Do R. K., Sali A., Protein Sci., 9, 1753-1773 (2000).

18) Jorgensen W. L., Chandrasekhar J., Madura J. D., Impey R. W., Klein M. L., J. Chem. Phys., 79, 926-935 (1983).

19) Case D. A., Cheatham T. E. 3rd, Darden T., Gohlke H., Luo R., Merz K. M. Jr., Onufriev A., Simmerling C., Wang B., Woods R. J., J. Comput. Chem., 26, 1668-1688 (2005).

20) Pearlman D. A., Case D. A., Caldwell J. W., Ross W. S., Cheatham T. E., Debolt S., Ferguson D., Seible G., Kollman P., Comput. Phys. Commun., 91, 1-41 (1995).

21) Brooks B. R., Brooks C. L. III, Mackerell A. D., Nilsson L., Petrella R. J., Roux B., Won Y., Archontis G., Bartels C., Boresch S., Caflisch A., Caves L., Cui Q., Dinner A. R., Feig M., Fischer S., Gao J., Hodoscek M., Im W., Kuczera K., Lazaridis T., Ma J., Ovchinnikov V., Paci E., Pastor R. W., Post C. B., Pu J. Z., Schaefer M., Tidor B., Venable R. M., Woodcock H. L., Wu X., Yang W., York D. M., Karplus M., J. Comput. Chem., 4, 187-217 (1983).

22) Pastor R. W., Brooks B. R., Szabo A., Mol. Phys., 65, 1409-1419 (1988).

23) Loncharich R. J., Brooks B. R., Pastor R. W., Biopolymers, 32, 523 535 (1992).

24) Izaguirre J. A., Catarello D. P., Wozniak J. M., Skeel R. D., J. Chem. Phys., 114, 2090-2098 (2001).

25) Ryckaert J. P., Ciccotti G., Berendsen H. J. C., J. Comput. Phys., 23, 327-341 (1977).

26) Kollman P., Chem. Rev., 93, 2395-2417 (1993).

27) Golhke H., Case D. A., J. Comput. Chem., 25, 238-250 (2004).

28) Manning T. C., Schlueter C. J., Brodnicki T. C., Parke E. A., Speir J. A., Garcia K. C., Teyton L., Wilson I. A., Kranz D. M., Immunity, 8, 413-425 (1998).

29) Zoete V., Michielin O., Proteins, 67, 1026-1047 (2007).

30) Chelvanayagam G., Immunogenetics, 45, 15-26 (1996).

31) Rammensee H., Bachmann J., Emmerich N. P., Bachor O. A., Stevanovic S., Immunogenetics, 50, 213-219 (1999).

32) Teeter M. M., Case D. A., J. Phys. Chem., 94, 8091-8097 (1990).

33) Kitao A., Go N., Curr. Opin. Struct. Biol., 9, 164-169 (1999).

34) Zeh H. J. III, Leder G. H., Lotze M. T., Salter R. D., Tector M., Stuber G., Modrow S., Storkus W. J., Hum. Immunol., 39, 79-86 (1994).

35) DeLano W. L., The DeLano Scientific, San Carlos, CA, U.S.A., 2002

36) Madden D., Garboczi D., Wiley D., Cell, 75, 693-708 (1993).

37) Fujiwara M., Tanuma J., Koizumi M., Kawashima Y., Honda K., Matsuoka-Aizawa S., Dohki S., Oka S., Takiguchi M., J. Virol., 82, $138-147$ (2008)

38) Tsurui H., Takahashi T., J. Pharmacol. Sci., 105, 299-316 (2007).

39) Antes I., Siu S. W., Lengauer T., Bioinformatics, 22, e16-e24 (2006).

40) Flower D. R., Phadwal K., Macdonald I. K., Coveney P. V., Davies M. N., Wan S., Immunome Res., 6 (Suppl. 2), S4 (2010).

41) Lim J. S., Kim S., Lee H. G., Lee K. Y., Kwon T. J., Kim K., Mol. Immunol., 33, 221-230 (1996).

42) Pöhlmann T., Böckmann R. A., Grubmüller H., Uchanska-Ziegler B., Ziegler A., Alexiev U., J. Biol. Chem., 279, 28197-28201 (2004).

43) Starikov E. B., Nilsson L., Hülsmeyer M., Eur. Biophys. J., 33, 651655 (2004).

44) Stavrakoudis A., Tsoulos I. G., Uray K., Hudecz F., Apostolopoulos V., J. Mol. Model., 17, 1817-1829 (2011).

45) Knapp B., Omasits U., Schreiner W., Epstein M. M., PLoS ONE, 5, e11653 (2010).

46) Painter C. A., Cruz A., López G. E., Stern L. J., Zavala-Ruiz Z., PLoS ONE, 3, e2403 (2008).

47) Nojima H., Takeda-Shitaka M., Kurihara Y., Adachi M., Yoneda S., Kamiya K., Umeyama H., Chem. Pharm. Bull., 50, 1209-1214 (2002).

48) Nojima H., Takeda-Shitaka M., Kurihara Y., Kamiya K., Umeyama H., Chem. Pharm. Bull., 51, 923-928 (2003).

49) Nojima H., Takeda-Shitaka M., Kanou K., Kamiya K., Umeyama H., Chem. Pharm. Bull., 56, 635-641 (2008). 
50) Nojima H., Kanou K., Kamiya K., Atsuda K., Umeyama H., TakedaShitaka M., Chem. Pharm. Bull., 57, 1193-1199 (2009).

51) Tang Y., Lin Z., Ni B., Wei J., Han J., Wang H., Wu Y., Cancer Immunol. Immunother, 56, 319-329 (2007).

52) Joseph M. A., Mitchell M. L., Evanseck J. D., Kovacs J. R., Jia L., Shen H., Meng W. S., Mol. Immunol., 44, 322-331 (2007).

53) Toh H., Savoie C. J., Kamikawaji N., Muta S., Sasazuki T., Kuhara S., Biopolymers, 54, 318 - 327 (2000).

54) Toh H., Kamikawaji N., Tana T., Sasazuki T., Kuhara S., Protein Eng., 11, 1027-1032 (1998).

55) Fabian H., Huser H., Narzi D., Misselwitz R., Loll B. Zieglery, Böckmann RA., Uchanska-Ziegler B., Naumann D., J. Mol. Biol., 376, $798-810$ (2008)
56) Sieker F., Straatsma T. P., Springer S., Zacharias M., Mol. Immunol., 45, 3714-3722 (2008)

57) Sieker F., Springer S., Zacharias M., Protein Sci., 16, 299-308 (2007).

58) Todman S. J., Halling-Brown M. D., Davies M. N., Flower D. R., Kayikci M., Moss D. S., J. Mol. Graph. Model., 26, 957-961 (2008).

59) Fagerberg T., Cerottini J. C., Michielin O., J. Mol. Biol., 356, 521$546(2006)$

60) Cárdenas C., Bidon-Chanal A., Conejeros P., Arenas G., Marshall S., Luque F. J., J. Comput. Aided Mol. Des., 24, 1035-1051 (2010).

61) Wan S., Coveney P. V., Flower D. R., J. Immunol., 175, 1715-1723 (2005). 